Canadian

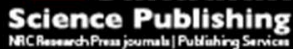

Applied Physiology, Nutrition, and Metabolism Physiologie appliquée, nutrition et métabolisme

\title{
The impact of moderate altitude on exercise metabolism in recreational sportsmen: a nuclear magnetic resonance metabolomic approach
}

\begin{tabular}{|r|l|}
\hline Journal: & Applied Physiology, Nutrition, and Metabolism \\
\hline Manuscript ID & apnm-2016-0717.R2 \\
\hline Manuscript Type: & Article \\
\hline Date Submitted by the Author: & 23-May-2017 \\
\hline Complete List of Authors: & $\begin{array}{l}\text { Messier, Florian; Universite de Perpignan, Laboratoire Européen } \\
\text { Performance Santé Altitude } \\
\text { Le Moyec, Laurence; Universite d'Evry-Val-d'Essonne } \\
\text { Santi, Carole; Universite de Perpignan } \\
\text { Gaston, Anne-Fleur; Universite de Perpignan } \\
\text { Triba, Mohamed N; Universite Paris 13 Campus de Bobigny } \\
\text { Roca, Emma ; Universitat de Girona } \\
\text { Durand, Fabienne; Universite de Perpignan }\end{array}$ \\
\hline Is the invited manuscript for \\
consideration in a Special \\
Issue? :
\end{tabular}


The impact of moderate altitude on exercise metabolism in recreational sportsmen: a nuclear magnetic resonance metabolomic approach

Florian M Messier ${ }^{\mathrm{a},}$, Laurence Le Moyec ${ }^{\mathrm{b}}$, Carole Santi ${ }^{\mathrm{a}}$, Anne-Fleur Gaston ${ }^{\mathrm{a}}$, Mohamed N. Triba ${ }^{\mathrm{c}}$, Emma Roca $^{\mathrm{a}, \mathrm{d}}$, Fabienne Durand ${ }^{\mathrm{a}}$

${ }^{a}$ Laboratoire Européen Performance Santé Altitude EA4604 - Université de Perpignan Via Domitia, Département STAPS, 7 Avenue Pierre de Coubertin, Font-Romeu, France

${ }^{\mathrm{b}}$ Unité de Biologie Intégrative et Adaptation à l'Exercice, EA 7362, Université d'Evry Val D'Essonne, Evry, France

${ }^{\mathrm{c}}$ Chimie Structures Propriétés de Biomatériaux et d'Agents Thérapeutiques, CNRS UMR 7244, Université Paris 13 Sorbonne Paris Cité, Bobigny, France

${ }^{\mathrm{d}}$ Facultat de Medicina, Universitat de Girona, C/ Emili Grahit 77, Girona, Spain

*Corresponding author:

Tel.: + 33468308074

E-mail address: florian.messier@univ-perp.fr 


\begin{abstract}
$\underline{\text { Abstract }}$
Although it is known that altitude impairs performance in endurance sports, there is no consensus on the involvement of energy substrates in this process. The objective of the present study was to determine whether the metabolomic pathways used during endurance exercise differ according to whether the effort is performed at SL or at moderate ALT (at the same exercise intensity, using proton nuclear magnetic resonance, ${ }^{1} \mathrm{H}$ NMR). Twenty subjects performed two 60-min endurance exercise tests at sea level and at $2150 \mathrm{~m}$ at identical relative intensity on a cycle ergometer. Blood plasma was obtained from venous blood samples drawn before and after exercise. Proton NMR spectral analysis was then performed on the plasma samples. A multivariate statistical technique was applied to the NMR data. The respective relative intensities of the sea level and altitude endurance tests were essentially the same when expressed as a percentage of the $\mathrm{VO}_{2} \mathrm{max}$ measured during the corresponding incremental maximal exercise test. Lipid use was similar at sea level and at altitude. In the plasma, levels of glucose, glutamine, alanine and branched-chain amino acids had decreased after exercise at altitude but not after exercise at sea level. The decrease in plasma glucose and free amino acid levels observed after exercise at altitude indicated that increased involvement of the protein pathway was necessary but not sufficient for the maintenance of glycaemia. Metabolomics is a powerful means of gaining insight into the metabolic changes induced by exercise at altitude.
\end{abstract}

Keywords: Altitude, exercise, metabolomics, nuclear magnetic resonance, plasma, metabolism. 


\section{Introduction}

It is well known that altitude impairs performance in endurance sports, despite the presence of many interindividual differences related to the magnitude of the altitude, the degree of acclimatization, endurance training status and probably other parameters that have yet to be identified (Keys et al. 1936, Gore et al. 1996, Mollard et al. 2007). Although performance is the result of a complex combination of physiological parameters, substrate use has a major role in endurance sport.

Several groups of researchers have investigated substrate use at high altitudes (3000-4300 m) or during severe hypoxia at sea level (SL); however, these literature data are contradictory. At the same absolute work rate, carbohydrate use was reportedly higher during exercise at high altitude $(4300 \mathrm{~m})$ than during exercise at SL (Brooks et al. 1991, Roberts et al. 1996). In contrast, a few studies have observed that high altitude or severe hypoxia has no influence on carbohydrate use (Bouissou et al. 1987, Lundby and Van Hall 2002). However, the interpretation of these findings is complicated by the use of a higher relative intensity of exercise at altitude, which would tend to increase reliance on glucose as a fuel (Brooks and Mercier 1994, Beidleman et al. 2002, Mazzeo 2008). During exercise at altitude, carbohydrate might contribute more to the total energy yield because free fatty acid oxidation requires more oxygen per ATP molecule synthetized than the complete oxidation of carbohydrates (Hinkle et al. 1991).

Despite the fact that many popular endurance sports (such cross-country skiing, ski mountaineering and trail running) are performed at moderate altitudes (i.e. at between 2000 and 3000 meters above SL), few studies have been performed in this setting. Furthermore, most sports training camps take place located at moderate altitude. In fact, substrate use at moderate altitude has not been extensively investigated. The results of a study by Katayama et al. (2010) suggested that carbohydrate use is higher during exercise at $50 \%$ of $\mathrm{VO}_{2}$ max performed at a simulated altitude of $2000 \mathrm{~m}$ than the same exercise during normoxia (at the same relative intensity). Furthermore, the intensity applied by Katayama et al. (2010) $\left(50 \%\right.$ of $\left.\mathrm{VO}_{2} \mathrm{max}\right)$ is also relatively low for endurance exercise in the field. Indeed, it is well known that the intensity of endurance training is usually situated between the first ventilatory threshold (VT1, i.e. $40-60 \%$ of $\mathrm{VO}_{2} \mathrm{max}$ ) and the second first ventilatory threshold (i.e. $60-90 \%$ of $\mathrm{VO}_{2} \max$ ) (Hawley et al. 1997).

Another point relates to the small number of key metabolites assayed in the majority of studies seeking to test specific biochemical hypotheses (Braun et al. 2000, Katayama et al. 2010). It is well known that metabolic pathways such as glycolysis and lipolysis interacted significantly through a large number of metabolites. Hence, we hypothesized that gaining a comprehensive view of the metabolome and its modulation by exercise at moderate altitude might help to reveal the underlying mechanism and novel (possibly unexpected) markers of performance and metabolic adaptation to hypoxia. This approach is possible with metabolomic techniques, i.e. the identification and quantification of a large set of metabolites present in living organisms (Wilson 2008). Recent research on human subjects and animal models has generated fascinating metabolomic data on strenuous endurance exercise (Pohjanen et al. 2007, Lewis et al. 2010), strength-endurance training (Yan et al. 2009), vigorous exercise, the effect of a specific diet on exercise performance (Kirwan et al. 2009) and the effect of the post-exercise ingestion of rehydration drinks (Chorell et al. 2009, Miccheli et al. 2009). Most recently, a 
metabolomic study by Lou et al. (2014) found changes in energy pathways (lipid metabolism) at altitudes of $3000-4000 \mathrm{~m}$ in six healthy, sedentary men. Along with mass spectrometry, nuclear magnetic resonance (NMR) is the main analytical tool used for metabolite fingerprinting (Lenz and Wilson 2007). NMR also has major advantages for untargeted metabolite profiling, including robustness, identification power, repeatability and reproducibility (Dumas et al. 2006).

In this context, the objective of the present study was to determine whether the metabolomic pathways used during endurance exercise differ according to whether the effort is performed at SL or at moderate ALT (at the same exercise intensity).We used ${ }^{1} \mathrm{H}$ NMR spectroscopy to determine the metabolomic profiles of plasma samples from recreational sportsmen and assessed the profiles' relationship with cardiorespiratory data. We hypothesised that because the partial pressure of inspired oxygen $\left(\mathrm{O}_{2}\right)$ falls with altitude, endurance exercise at moderate altitude would elicit greater metabolic disturbances (i.e. a greater pre- vs. post- exercise difference in the | metabolomic profile) than the same relative exercise intensity at sea level. 


\section{Methods}

Participants

Twenty healthy male volunteers were included in the study. The mean \pm standard deviation (SD) age, height and body mass were respectively $39 \pm 4.3$ years, $178 \pm 5.3 \mathrm{~cm}$, and $76 \pm 9.0 \mathrm{~kg}$. The mean body mass index was 24.3 \pm 2.3 , and the mean fat mass was $18.9 \pm 3.6 \%$. All the study participants lived at SL and participated in educational or recreational physical activities at low to moderate intensities, in most cases. The participants trained for an average of $9 \pm 3$ hours per week and have a mean $\mathrm{VO}_{2}$ max (measured at SL) of $53 \pm 8 \mathrm{ml}^{\mathrm{min}} \mathrm{mig}^{-1}$

1 . None of the participants were smokers, and none were taking medications or vitamin supplements. All were free of infections and cardiopulmonary conditions that would have contraindicated exercise. All the volunteers were informed about the experimental procedures and gave their written, informed consent to participation. The study protocol was approved by the local independent ethics committee (Consell General de l'Esport, Catalunya, Spain).

\section{Study design}

In order to control for the effect of nutrition on substrate use during exercise, the participants were told to follow a standardized diet on the day preceding the exercise tests. The dietary plan provided $55 \%$ of the energy as carbohydrate, $30 \%$ as fat, and $15 \%$ as protein. On the morning before exercise testing, the participants were given a standardized meal. All participants performed a test on an ergometer cycle (Racer 9, Kettler, Germany) under two distinct conditions: first at SL and then (two weeks later) at an altitude 2150 meters (ALT).

Firstly, an incremental maximal exercise test was performed in order to determine performance parameters in general and VT1 in particular, as described by Wasserman et al. 1973. The exercise started with a 3 min warm-up period at $60 \mathrm{~W}$ and a pedalling rate of $70 \mathrm{rpm}$. The workload was then increased by $30 \mathrm{~W}$ every minute until exhaustion. The test was considered to be maximal if at least three of the following criteria were met: (i) an increase in $\mathrm{VO}_{2}$ of $<100 \mathrm{ml}$ for the last increment in the work rate, (ii) achievement of the age-predicted maximal heart rate $(\mathrm{HR})$ [210-(0.65 age) $\pm 10 \%]$, (iii) a respiratory exchange ratio (RER) above 1.1 , and (iv) inability to maintain the imposed pedalling rate despite maximal effort and verbal encouragement.

Secondly, $24 \mathrm{~h}$ after the incremental maximal exercise test, participants performed a 60-minute endurance exercise test at the previously determined VT1. The endurance test began at $60 \mathrm{~W}$ for 3 minutes. The intensity was then increased by $30 \mathrm{~W}$ every minute until the participant's individual VT1 was reached. The pedalling rate was kept constant at $70 \mathrm{rpm}$. During each test, cardiorespiratory parameters and $\mathrm{O}_{2}$ saturation were continuously recorded.

During the altitude session, the participants arrived at altitude 90 minutes before the maximal effort test (i.e. in the absence of an acclimatization phase). Between the maximal test and the endurance test, the participants were lodged at an altitude of around $1000 \mathrm{~m}$. They then climbed to an altitude of $2150 \mathrm{~m} 90$ minutes before the endurance test. The time interval between the maximum test and the endurance test was never more than 36 hours. 
Cardiorespiratory parameters

A breath-by-breath telemetric, portable gas analyzer (K4b2, Cosmed, Rome, Italy) was used to collect the gas exchange data for all tests: oxygen uptake $\left(\mathrm{VO}_{2}, \mathrm{ml} \cdot \mathrm{min}^{-1} \cdot \mathrm{kg}^{-1}\right), \mathrm{RER}\left(\mathrm{VCO}_{2} / \mathrm{VO}_{2}\right)$, minute ventilation (VE, 1.min ${ }^{1}$ ), end-tidal carbon dioxide pressure $(\mathrm{PetCO}, \mathrm{mmHg})$, and ventilatory equivalents $\left(\mathrm{VE} / \mathrm{VO}_{2}\right.$ and $\left.\mathrm{VE} / \mathrm{VCO}_{2}\right)$. Before every test session, the gas analyzer was calibrated according to the manufacturer's specifications, using a 3-1 syringe and a gas bottle of known $\mathrm{O}_{2}$ and $\mathrm{CO}_{2}$ concentrations (16\% and $5 \%$, respectively). Throughout the test, each participant was equipped with a chest belt (Polar Electro, Kempele, Finland) in order to continuously record the HR (beats. $\left.\min ^{-1}\right)$. Blood haemoglobin $\mathrm{O}_{2}$ saturation $\left(\mathrm{SpO}_{2}\right)$ was measured throughout the tests with an oximeter (Nonin Medical Inc., Plymouth, MN, USA) equipped with an ear sensor.

\section{Blood samples}

Blood samples were collected from the median cubital vein (in the cubital fossa anterior to the elbow) in a $7 \mathrm{~mL}$ lithium-heparin tube before (T0) each endurance exercise test session and after $60 \mathrm{~min}$ at VT1 (T60). At the blood sampling time point at T60, the participants were still pedalling (in order to maintain their effort at VT1). Plasma was separated by centrifugation for $10 \mathrm{~min}$ at $2500 \mathrm{~g}$ and $4^{\circ} \mathrm{C}$. The plasma samples were divided into aliquots and stored at $-80^{\circ} \mathrm{C}$ until analysis.

${ }^{1} \mathrm{H}$ NMR spectroscopy analysis

Plasma samples were thawed at room temperature. $450 \mu \mathrm{L}$ of plasma were placed in a $5 \mathrm{~mm}$ NMR tube, along with $100 \mu \mathrm{L}$ of deuterium oxide (for field locking). The proton spectra were acquired at $300 \mathrm{~K}$ on a $500 \mathrm{MHz}$ Avance III spectrometer (Advance III spectrometer, Bruker, Germany) with a $5 \mathrm{~mm}$ reversed QXI Z gradient high resolution probe (Bruker). Free induction decays (FIDs) were acquired using a one-dimensional nuclear Overhauser effect spectroscopy (1D-NOESY) sequence for water suppression, with a 2 s pre-acquisition delay, a $100 \mathrm{~ms}$ mixing time and a $90^{\circ}$ pulse. The FIDs were collected as $32 \mathrm{~K}$ complex points in a spectral window of $10000 \mathrm{~Hz}$ and 64 transients after four silent scans. The use of a 1D-NOESYsequence does not interfere with the lipid resonance. The FIDs were processed with NMRpipe software. The exponential Fourier transform produced a $0.3 \mathrm{~Hz}$ line broadening. Spectra were phased, and a 10-point baseline correction was applied. Each spectrum was calibrated using the alanine signal at $1.48 \mathrm{ppm}$. The spectral region between $-1 \mathrm{ppm}$ and $11 \mathrm{ppm}$ was divided into 12000 spectral buckets (width: $0.001 \mathrm{ppm}$ ), using an in-house MATLAB script. The water region (between 4.6 and $5.15 \mathrm{ppm}$ ) was excluded. Probabilistic quotient normalization was used to obtain the $\mathrm{X}$ matrix for statistical analysis (Dieterle et al. 2006) and correct for concentration effects related to possible dehydration after the race. All variables were autoscaled before multivariate statistical analysis.

\section{Statistical analysis}

To compare the plasma spectra at $\mathrm{T} 0$ and $\mathrm{T} 60$, models were computed with a paired set of samples from the same participant at SL and at ALT, as described by Westerhuis et al. (2010). Principal component analysis (PCA) and supervised multivariate statistical analysis were performed using in-house MATLAB scripts (Mathworks, Natick, 
MA) based on the Trygg and Wold (2002) method, as previously described (Nahon et al. 2012). The PCA was first performed with the X matrix, in order to detect any group separation (based on NMR signal variability and possible outliers). A supervised multivariate statistical technique (orthogonal projection to latent structure (OPLS) analysis was applied to the NMR data. The model's quality was assessed with regard to the correlation coefficient $\left(R^{2} Y\right)$ and the predictive coefficient $\left(Q^{2} Y\right): R^{2} Y=1$ indicates perfect description of the data by the model, whereas $Q^{2} Y=1$ indicates perfect predictability. $Q^{2} Y$ was computed with the "leave-one-out" cross-validation method (N. Triba et al. 2015). A 999-permutation test was also used for internal validation of the OPLS models, i.e. whether the OPLS models built with the samples were significantly better than any other OPLS model obtained by random permutation of the original sample's attributes.

A score plot and a loading plot were used to illustrate the results. In the score-plot, each point represents the projection of an NMR spectrum (and thus a plasma sample) on to the model's predictive component (Tpred, horizontal axis) and first orthogonal component (Torth, vertical axis). The loading plot represents the covariance between the Y-response matrix and the signal intensity of the various spectral domains. Colours were also used in the loading plot, depending on the $\mathrm{R}$ value for the correlation between the corresponding bucket intensity and the $\mathrm{Y}$ variable. Metabolites were considered as to be discriminant when they corresponded to buckets with $\mathrm{R} \geq 0.5$. Spectral regions were then investigated, in order to identify the metabolites responsible for the classification. Metabolites were identified by using the Human Metabolome Database (University of Alberta, Canada; Wishart 2007).

A paired t-test or Wilcoxon's test was used to analyse SL vs. ALT differences in cardiorespiratory parameters. For all tests, the threshold for statistical significance was set to $\mathrm{p}<0.05$. Analyses were conducted using SigmaStat software (version 3.5, Systat Software Inc., San José, CA, USA). Data are quoted as the mean \pm SD.

\section{Results}

\section{Cardiorespiratory parameters}

Analysis of the incremental maximal exercise tests revealed a lower $\mathrm{VO}_{2} \max$ and lower peak power at ALT, relative to SL ( $46 \pm 7$ vs. $53 \pm 8 \mathrm{ml} \cdot \mathrm{min}^{-1} \cdot \mathrm{kg}^{-1}$, respectively, $\mathrm{p}<0.05$; and $376 \pm 53$ vs. $401 \pm 61 \mathrm{~W}$, respectively, $\mathrm{p}<0.05)$. The ALT vs SL difference in $\mathrm{VO}_{2}$ max was $-13 \pm 3 \%$. The RER during maximal exercise was above 1.1 in each condition and was higher at ALT than at SL $(1.26 \pm 0.07$ vs $1.15 \pm 0.04$, respectively; $\mathrm{p}<0.05)$.

Performance values measured during the endurance exercise test, at SL and at ALT are reported in Table 1. $\mathrm{VO}_{2}$, power output, $\mathrm{SpO}_{2}$ and $\mathrm{HR}$ at 60 min were significantly lower at ALT than at $\mathrm{SL}$ - even though the test's relative intensity (expressed as a percentage of $\mathrm{VO}_{2}$ max measured during the corresponding maximal exercise test) was the same in both settings. $\mathrm{VE} / \mathrm{VCO}_{2}$ and RER were higher at ALT than at $\mathrm{SL}(\mathrm{p}<0.05)$. VE was essentially the same at ALT and at SL. 
Metabolomic results

Figure 1 represents the PCA applied to data after the endurance tests (T0) at SL and at ALT. The PCA probed the homogeneity of the subject's metabolic profile before the test at SL and at ALT. The model was not able to discriminate between SL T0 samples and ALT T0 samples, which indicated than the participants' metabolic profiles were homogeneous before exercise at SL and at ALT. However, two of the samples (an SL sample and an ALT sample for the same participant) were clearly outliers. This participant had a particular diet, including a high proportion of oil-containing foods (such as olives, seeds and nuts). This participant was excluded from subsequent analyses.

The spectra of plasma samples obtained at T0 and T60 at SL were first compared using the paired method. The PCA demonstrated that the SL samples were homogeneous, albeit with a slight separation between T0 samples and T60 samples (Fig 2a). In a comparison of the T0 and T60 spectra at SL, the OPLS model (Fig 2b) displayed good statistical parameters $\left(R^{2} Y=0.977\right.$ and $\left.Q^{2} Y=0.726\right)$. The permutation process validated this model, since none of Q2T values obtained by permutation was higher than the Q2Y value obtained with non-permuted data. The loading plot (Fig 2c) indicated the correlation between the spectral regions and prediction axis. The spectral regions with a correlation over $|0.5|$ are listed in Table 2, along with their assignments to metabolites. These regions correspond mainly to the glycerol signal (which was higher at T60 than at T0) and the lipid signal (which was lower at T60 than at T0). Likewise, the PCA demonstrated that the ALT samples were homogeneous, albeit with a slight separation between T0 samples and T60 samples (Fig 3a). In a comparison of the T0 and T60 spectra at ALT, the OPLS model (Fig 3b) displayed good statistical parameters $\left(R^{2} Y=0.990\right.$ and $Q^{2} Y=0.756$ ). Again, the permutation process validated the model. The loading plot (Fig 3c) indicated a correlation between the spectral regions and the prediction axis. The spectral regions with a correlation over $|0.5|$ are listed in Table 2, along with their assignments to metabolites. These regions corresponded mainly to glycerol and lactate signals (which were higher at T60 than at T0) and to lipid, glucose, glutamine, alanine and branched-chain amino acid (BCAA) signals (which were lower at T60 than at T0). 


\section{Discussion}

The objective of the present study was to determine whether the metabolomic pathways used during endurance exercise differed according to whether the effort is performed at SL or at moderate ALT (at the same exercise intensity).

${ }^{1} \mathrm{H}$ NMR spectroscopy is an efficient, robust, cost-effective metabolomics technique for acquiring metabolic profiles without the need for extensive sample preparation. Here, application of a metabolomic method revealed changes in the three main metabolic pathways (carbohydrate, lipid and protein pathways) induced by exercise at moderate ALT (relative to SL). Previous research (using biochemical methods) had shown partial modulation of these pathways, whereas NMR-based metabolomics simultaneously harvested information on various metabolites in a single spectrum (Gao et al. 2008) - thus facilitating the rapid identification of the metabolic pathways involved in exercise. With regard to inter-individual variations in metabolic profiles during exercise, it is very well known that diet has a major effect (Chorell et al. 2009, Miccheli et al. 2009, Kirwan et al. 2009). In the present study, we reduced (but did not eliminate) inter-individual variability by asking the participants to follow a standardized diet for 24 hours before the exercise sessions and on the day of the tests. We therefore used a paired sample approach to model the data; each participant acted as his own control, thus minimizing intra-individual variations (Westerhuis et al. 2010). Maximal exercise tests at SL and ALT were used to determine VT1, allowing us to minimize bias related to the exercise intensity at SL and at ALT. Our data confirmed that the same degree of physiological stress was present during endurance exercise at SL and ALT, with a similar relative intensity (69\% of $\mathrm{VO}_{2}$ max at $\mathrm{SL}$ vs $70 \%$ at $\mathrm{ALT}$, for $\mathrm{VO}_{2}$ max measured during the incremental maximal exercise test). Our tests at SL and at ALT were not performed in random order. We are aware that the lack of randomization may have influenced test learning. However, the study participants were active recreational athletes who were already familiar with endurance cycling. Given that the ALT test was truly performed at altitude (and not at SL under hyperbaric conditions), logistic constraints prevented us from using the same equipment for exercise sessions and measurements at both SL and at ALT.

As has also been described in the literature, $\mathrm{VO}_{2}$, power output and $\mathrm{SpO}_{2}$ during exercise were significantly lower at ALT than at SL (Gore et al. 1996, Gaston et al. 2016). While no difference was observed for the VE during exercise between SL and ALT, VE/VCO2 was higher and $\mathrm{PetCO}_{2}$ was lower at ALT when compared to SL suggesting excessive hyperventilation and poor ventilatory efficiency (Mollard et al. 2007). The RER measured at T60 was greater at ALT than at SL, which is suggestive of greater glucose use at moderate ALT. Nevertheless, the RER may have been influenced by several parameters, such as the pre-test diet and overnight fasting (Jansson 1982, Beidleman et al. 2002). This bias was minimized in the present study because participants ate the same diet for $24 \mathrm{~h}$ before each test. Secondly, the RER value depends on the accuracy of indirect calorimetry measurements during exercise. Hyperventilation may lead to the overestimation of tissue $\mathrm{CO}_{2}$ production (Ferrannini 1988). The elevated $\mathrm{VE} / \mathrm{VCO}_{2}$ at $\mathrm{T} 60$ in the ALT test was suggestive of this type of over-estimation. Indeed, this finding agrees with Katayama et al.'s (2010) report of higher RER values during exercise with moderate, normobaric hypoxia (simulating an ALT of $2000 \mathrm{~m}$ ). In contrast, studies performed at higher ALTs (Lundby and Van Hall 
2002 and Bouissou et al. 1987) did not observe any difference in the RER during exercise at the same relative intensity ( 50 or $60 \%$ of $\mathrm{VO}_{2} \mathrm{max}$ ), when compared with SL.

The NMR metabolomic profiles described above showed that metabolic adaptations to endurance exercise differed at SL and at ALT. Hence, these results confirmed our hypothesis whereby metabolic energy supply pathways differ at SL vs. ALT. The main differences concerned glucose and free amino acid levels but not lipid. Indeed, at the same relative exercise intensity (VT1) at SL and at ALT, lipid use was similar but glucose, glutamine, alanine and BCAA levels were lower in the metabolome profile at T60 at ALT. These metabolites were not discriminant in the OPLS model calculated for the SL test. Likewise, lactate was elevated at ALT perhaps due (at least in part) to increased glycolysis, as suggested by Katayama et al. (2010).

It is well known that lipids are consumed during moderate endurance exercise (Brooks and Mercier 1994, Horowitz and Klein 2000). An increase in glycerol content results from the release of free fatty acids from triglycerides. Our data did not reveal an SL vs. ALT difference in lipid metabolism. This is in agreement with literature data suggesting that at equivalent relative workloads (as a percentage of $\mathrm{VO}_{2} \mathrm{max}$ ), lipid substrate use is unchanged during acute and chronic hypoxia (Lundby and Van Hall 2002).

According to the metabolomic profiles, glycaemia fell during the test at ALT but not at SL. In the present study, the RER measured at T60 was also higher at ALT than at SL. Taken as a whole, our results suggest that glucose utilization is greater at ALT. Likewise, Katayama et al. (2010) showed that exercise at moderate relative intensity $\left(50 \%\right.$ of $\left.\mathrm{VO}_{2} \mathrm{max}\right)$ at simulated moderate ALT $(2000 \mathrm{~m})$ increased carbohydrate use. At higher ALT (4100 m), Lundby and Van Hall (2002) reported that glucose metabolism is the same as at sea level. The ALT tested in our study (i.e. 2150 meters) may have been associated with significant levels of hypoxic cellular stress and greater use of glycolytic fibres during exercise - resulting in a fuel shift from lipid oxidation to carbohydrate oxidation. During exercise at ALT, it seems that stable glycaemia could not be maintained by lipid oxidation.

Our metabolomic approach assessed the three main metabolic pathways. The participants' metabolomic profiles also demonstrated that protein pathways were involved in exercise at ALT but not at SL. At SL, Bergström et al. (1985) have found that BCAA muscle concentrations were the same before and after exercise at $50-70 \%$ of $\mathrm{VO}_{2}$ max. At ALT, our results agree with Bigard et al.'s (1993) report that repeated, daily sessions of longduration exercise induced a significant alteration in the plasma amino acid pattern. The lower plasma BCAA, glutamine and alanine levels after exercise at ALT suggest that enhanced proteolysis and/or energy substrate supplementation are required to maintain glycaemia (Levin et al. 1983). Shimizu et al. (2011) have shown that BCAA supplementation has a moderate but useful positive effect on fatigue when trekking at $2750 \mathrm{~m}$. Furthermore, it has been suggested that the decrease in plasma BCAA concentration is one of the causative factors for fatigue during prolonged exercise (Parry-Billings et al. 1990). The potential benefits of dietary BCAA supplementation on improving performance at moderate ALT warrant further investigation. 


\section{Conclusion}

Our present results demonstrated that at similar exercise intensity, substrate use during endurance exercise differed between SL and moderate ALT. The metabolic response to exercise was investigated with ${ }^{1} \mathrm{H}$ NMR spectroscopy of blood plasma samples. It appears that BCAAs were used to maintain glycaemia during endurance exercise at moderate ALT. Further studies should look at whether glucose and/or BCAA supplementation could prevent the fall in glycaemia during exercise at moderate altitude. In view of our present results for invasive (blood plasma) sampling, it would be interesting to see whether non-invasive sampling (e.g. urine sampling) might be of value in assessing human physiological adaptations to training and/or ALT. 


\section{Acknowledgements}

The authors give a special thanks to the subjects for their enthusiastic participation. We also acknowledge our appreciation to Girona Faculty of Medicine and Masella ski resort for their hospitality, Nadia Bouchemal (Université Paris 13) for NMR experiments and processing, Aurélie Collado, Anthony Sanchez and Florence Riera for their technical support.

\section{Conflict of interest}

The authors declare that they have no conflict of interest. 


\section{References}

Beidleman, B.A., Rock, P.B., Muza, S.R., Fulco, C.S., Gibson, L.L., Kamimori, G.H., and Cymerman, A. 2002. Substrate oxidation is altered in women during exercise upon acute altitude exposure: Med. Sci. Sports Exerc. 34(3): 430-437. doi:10.1097/00005768-200203000-00008.

Bouissou, P., Guezennec, C., Defer, G., and Pesquies, P. 1987. Oxygen Consumption, Lactate Accumulation, and Sympathetic Response During Prolonged Exercise Under Hypoxia. Int. J. Sports Med. 08(04): 266-269. doi:10.1055/s-2008-1025667.

Braun, B., Mawson, J.T., Muza, S.R., Dominick, S.B., Brooks, G.A., Horning, M.A., Rock, P.B., Moore, L.G., Mazzeo, R.S., Ezeji-Okoye, S.C., and others. 2000. Women at altitude: carbohydrate utilization during exercise at 4,300 m. J. Appl. Physiol. 88(1): 246-256.

Brooks, G.A., Butterfield, G.E., Wolfe, R.R., Groves, B.M., Mazzeo, R.S., Sutton, J.R., Wolfel, E.E., and Reeves, J.T. 1991. Increased dependence on blood glucose after acclimatization to 4,300 m. J. Appl. Physiol. 70(2): 919-927.

Brooks, G.A., and Mercier, J. 1994. Balance of carbohydrate and lipid utilization during exercise: the "crossover" concept. J. Appl. Physiol. 76(6): 2253-2261.

Chorell, E., Moritz, T., Branth, S., Antti, H., and Svensson, M.B. 2009. Predictive Metabolomics Evaluation of Nutrition-Modulated Metabolic Stress Responses in Human Blood Serum During the Early Recovery Phase of Strenuous Physical Exercise. J. Proteome Res. 8(6): 2966-2977. doi:10.1021/pr900081q.

Dieterle, F., Ross, A., Schlotterbeck, G., and Senn, H. 2006. Probabilistic Quotient Normalization as Robust Method to Account for Dilution of Complex Biological Mixtures. Application in 1H NMR Metabonomics. Anal. Chem. 78(13): 4281-4290. doi:10.1021/ac051632c.

Dumas, M.-E., Maibaum, E.C., Teague, C., Ueshima, H., Zhou, B., Lindon, J.C., Nicholson, J.K., Stamler, J., Elliott, P., Chan, Q., and Holmes, E. 2006. Assessment of Analytical Reproducibility of 1H NMR Spectroscopy Based Metabonomics for Large-Scale Epidemiological Research: the INTERMAP Study. Anal. Chem. 78(7): 2199-2208. doi:10.1021/ac0517085.

Ferrannini, E. 1988. The theoretical bases of indirect calorimetry: a review. Metabolism 37(3): $287-301$. 
Gao, H., Dong, B., Liu, X., Xuan, H., Huang, Y., and Lin, D. 2008. Metabonomic profiling of renal cell carcinoma: high-resolution proton nuclear magnetic resonance spectroscopy of human serum with multivariate data analysis. Anal. Chim. Acta 624(2): 269-277.

Gaston, A.-F., Durand, F., Roca, E., Doucende, G., Hapkova, I., and Subirats, E. 2016. Exercise-Induced Hypoxaemia Developed at Sea-Level Influences Responses to Exercise at Moderate Altitude. PLOS ONE 11(9): e0161819. doi:10.1371/journal.pone.0161819.

Gore, C.J., Little, S.C., Hahn, A.G., Scroop, G.C., Norton, K.I., Bourdon, P.C., Woolford, S.M., Buckley, J.D., Stanef, T., Campbell, D.P., Watson, D.B., and Emonson, D.L. 1996. Reduced performance of male and female athletes at $580 \mathrm{~m}$ altitude. Eur. J. Appl. Physiol. 75(2): 136-143. doi:10.1007/s004210050138.

Hawley, J.A., Myburgh, K.H., Noakes, T.D., and Dennis, S.C. 1997. Training techniques to improve fatigue resistance and enhance endurance performance. J. Sports Sci. 15(3): 325-333. doi:10.1080/026404197367335.

Hinkle, P.C., Kumar, M.A., Resetar, A., and Harris, D.L. 1991. Mechanistic stoichiometry of mitochondrial oxidative phosphorylation. Biochemistry (Mosc.) 30(14): 3576-3582.

Horowitz, J.F., and Klein, S. 2000. Lipid metabolism during endurance exercise. Am. J. Clin. Nutr. 72(2): 558s$563 \mathrm{~s}$.

Jansson, E. 1982. On the significance of the respiratory exchange ratio after different diets during exercise in man. Acta Physiol. Scand. 114(1): 103-110.

Katayama, K., Goto, K., Ishida, K., and Ogita, F. 2010. Substrate utilization during exercise and recovery at moderate altitude. Metabolism 59(7): 959-966.

Keys, A., Hall, F.G., and Barron, E.S.G. 1936. THE POSITION OF THE OXYGEN DISSOCIATION CURVE OF HUMAN BLOOD AT HIGH ALTITUDE. Am. J. Physiol. -- Leg. Content 115(2): 292-307.

Kirwan, G.M., Coffey, V.G., Niere, J.O., Hawley, J.A., and Adams, M.J. 2009. Spectroscopic correlation analysis of NMR-based metabonomics in exercise science. Anal. Chim. Acta 652(1-2): 173-179. doi:10.1016/j.aca.2009.07.011.

Lenz, E.M., and Wilson, I.D. 2007. Analytical Strategies in Metabonomics. J. Proteome Res. 6(2): $443-458$. doi:10.1021/pr0605217. 
Levin, L., Gevers, W., Jardine, L., De Guel, F.J.M., and Duncan, E.J. 1983. Serum amino acids in weight-losing patients with cancer and tuberculosis. Eur. J. Cancer Clin. Oncol. 19(6): 711-715. doi:10.1016/0277$5379(83) 90002-0$.

Lewis, G.D., Farrell, L., Wood, M.J., Martinovic, M., Arany, Z., Rowe, G.C., Souza, A., Cheng, S., McCabe, E.L., Yang, E., Shi, X., Deo, R., Roth, F.P., Asnani, A., Rhee, E.P., Systrom, D.M., Semigran, M.J., Vasan, R.S., Carr, S.A., Wang, T.J., Sabatine, M.S., Clish, C.B., and Gerszten, R.E. 2010. Metabolic Signatures of Exercise in Human Plasma. Sci. Transl. Med. 2(33): 33 ra37. doi:10.1126/scitranslmed.3001006.

Lou, B.-S., Wu, P.-S., Liu, Y., and Wang, J.-S. 2014. Effects of Acute Systematic Hypoxia on Human Urinary Metabolites Using LC-MS-Based Metabolomics. High Alt. Med. Biol. 15(2): 192-202. doi:10.1089/ham.2013.1130.

Lundby, C. e, and Van Hall, G. 2002. Substrate utilization in sea level residents during exercise in acute hypoxia and after 4 weeks of acclimatization to 4100 m. Acta Physiol. Scand. 176(3): 195-201.

Mazzeo, R.S. 2008. Physiological Responses to Exercise at Altitude. Sports Med. 38(1): 1-8. doi:10.2165/00007256-200838010-00001.

Miccheli, A., Marini, F., Capuani, G., Miccheli, A.T., Delfini, M., Di Cocco, M.E., Puccetti, C., Paci, M., Rizzo, M., and Spataro, A. 2009. The influence of a sports drink on the postexercise metabolism of elite athletes as investigated by NMR-based metabolomics. J. Am. Coll. Nutr. 28(5): 553-564.

Mollard, P., Woorons, X., Letournel, M., Lamberto, C., Favret, F., Pichon, A., Beaudry, M., and Richalet, J.-P. 2007. Determinants of maximal oxygen uptake in moderate acute hypoxia in endurance athletes. Eur. J. Appl. Physiol. 100(6): 663-673. doi:10.1007/s00421-007-0457-0.

Nahon, P., Amathieu, R., Triba, M.N., Bouchemal, N., Nault, J.-C., Ziol, M., Seror, O., Dhonneur, G., Trinchet, J.-C., Beaugrand, M., and Moyec, L.L. 2012. Identification of Serum Proton NMR Metabolomic Fingerprints Associated with Hepatocellular Carcinoma in Patients with Alcoholic Cirrhosis. Clin. Cancer Res. 18(24): 6714-6722. doi:10.1158/1078-0432.CCR-12-1099.

N. Triba, M., Moyec, L.L., Amathieu, R., Goossens, C., Bouchemal, N., Nahon, P., N. Rutledge, D., and Savarin, P. 2015. PLS/OPLS models in metabolomics: the impact of permutation of dataset rows on the K-fold cross-validation quality parameters. Mol. Biosyst. 11(1): 13-19. doi:10.1039/C4MB00414K. 
Parry-Billings, M., Blomstrand, E., McAndrew, N., and Newsholme, E. 1990. A Communicational Link Between Skeletal Muscle, Brain, and Cells of the Immune System. Int. J. Sports Med. 11(S 2): S122-S128. doi:10.1055/s-2007-1024863.

Pohjanen, E., Thysell, E., Jonsson, P., Eklund, C., Silfver, A., Carlsson, I.-B., Lundgren, K., Moritz, T., Svensson, M.B., and Antti, H. 2007. A Multivariate Screening Strategy for Investigating Metabolic Effects of Strenuous Physical Exercise in Human Serum. J. Proteome Res. 6(6): 2113-2120. doi:10.1021/pr070007g.

Roberts, A.C., Butterfield, G.E., Cymerman, A., Reeves, J.T., Wolfel, E.E., and Brooks, G.A. 1996. Acclimatization to 4,300-m altitude decreases reliance on fat as a substrate. J. Appl. Physiol. 81(4): $1762-1771$.

Westerhuis, J.A., van Velzen, E.J., Hoefsloot, H.C., and Smilde, A.K. 2010. Multivariate paired data analysis: multilevel PLSDA versus OPLSDA. Metabolomics 6(1): 119-128.

Wilson, N. 2008. Metabolomics, Metabonomics and Metabolite Profiling. Royal Society of Chemistry.

Wishart, D.S. 2007. Current Progress in computational metabolomics. Brief. Bioinform. 8(5): $279-293$. doi:10.1093/bib/bbm030.

Yan, B., A, J., Wang, G., Lu, H., Huang, X., Liu, Y., Zha, W., Hao, H., Zhang, Y., Liu, L., Gu, S., Huang, Q., Zheng, Y., and Sun, J. 2009. Metabolomic investigation into variation of endogenous metabolites in professional athletes subject to strength-endurance training. J. Appl. Physiol. 106(2): 531-538. doi:10.1152/japplphysiol.90816.2008. 
Table 1 Average performance data of the endurance exercise test between $20 \mathrm{~min}$ and $60 \mathrm{~min}$

\begin{tabular}{|c|c|c|}
\hline & \multicolumn{2}{|c|}{ Average between 20 and $60 \mathrm{~min}$} \\
\hline & Sea level & Altitude \\
\hline $\begin{array}{l}\mathrm{VO}_{2} \text { endurance test } \\
\left(\mathrm{ml} \cdot \mathrm{min} \cdot \mathrm{kg}^{-1}\right)\end{array}$ & $36 \pm 5$ & $31 * \pm 6$ \\
\hline $\begin{array}{l}\mathrm{VO}_{2} \max \\
(\%)\end{array}$ & $69 \pm 7$ & $67 \pm 8$ \\
\hline $\begin{array}{l}\text { Power Output } \\
\text { (Watts) }\end{array}$ & $203 \pm 34$ & $183^{*} \pm 31$ \\
\hline $\begin{array}{l}\text { VE } \\
\left(\mathrm{L} . \mathrm{min}^{-1}\right)\end{array}$ & $70 \pm 12$ & $71 \pm 9$ \\
\hline $\mathrm{VE} / \mathrm{VCO}_{2}$ & $27 \pm 4$ & $31^{*} \pm 4$ \\
\hline $\begin{array}{l}\text { Pet } \mathrm{CO}_{2} \\
(\mathrm{mmHg})\end{array}$ & $42 \pm 3$ & $36^{*} \pm 4$ \\
\hline $\begin{array}{l}\mathrm{SpO}_{2} \\
(\%)\end{array}$ & $98 \pm 4$ & $92 * \pm 2$ \\
\hline $\begin{array}{l}\text { HR } \\
\left(\text { beat.min }{ }^{-1} \text { ) }\right.\end{array}$ & $139 \pm 14$ & $130 * \pm 15$ \\
\hline RER & $0.92 \pm 0.06$ & $0.97^{*} \pm 0.05$ \\
\hline
\end{tabular}

* Significantly different from sea level $(\mathrm{P}<0.05) . \mathrm{n}=20$ in each group;

$\mathrm{VO}_{2}$ : Oxygen uptake; VE : Minute ventilation; Pet $\mathrm{CO}_{2}$ : End-tidal carbon dioxide pressure; $\mathrm{SpO}_{2}$ : $\mathrm{O} 2$ saturation of haemoglobin; HR : Heart rate; RER : Respiratory exchange ratio 
Table 2 List of peaks with correlation coefficient over 0.5 from the OPLS loading plot. Metabolites assignments and label in the loading plot. $r=$ correlation coefficient.

\begin{tabular}{|c|c|c|c|c|}
\cline { 4 - 5 } \multicolumn{2}{c}{} & \multicolumn{2}{c|}{ T0 Vs T60 } \\
shift & Metabolites & Labels & r plaine & r altitude \\
\hline 0.9 & $\mathrm{CH}_{3}$ fatty acids & 1 & -0.5 & -0.7 \\
\hline 0.96 & L-isoleucine & 2 & - & -0.8 \\
\hline 0.99 & L-leucine & 3 & - & -0.8 \\
\hline 1.04 & L-valine & 4 & - & -0.8 \\
\hline 1.29 & $\mathrm{CH}_{2}$ fatty acids & 5 & -0.5 & -0.7 \\
\hline 1.32 & Lactate & 6 & - & 0.6 \\
\hline 1.48 & Alanin & 7 & - & -0.6 \\
\hline 1.57 & $\mathrm{CH}_{2}$ fatty acids & 8 & -0.5 & -0.7 \\
\hline 2.01 & Fatty acids & 9 & -0.5 & -0.75 \\
\hline 2.12 & Glutamine & 10 & - & -0.7 \\
\hline 2.24 & Acetone & 11 & -0.55 & -0.55 \\
\hline 2.41 & Succinate & 12 & 0.8 & 0.8 \\
\hline 2.45 & Glutamine & 13 & - & -0.7 \\
\hline 3.57 & Glycerol & 14 & 0.9 & 0.9 \\
\hline 3.65 & Glycerol & 15 & 0.9 & 0.9 \\
\hline $3.0-4.0$ & Glucose & 16 & - & -0.5 \\
\hline 4.1 & Lactate & 17 & - & 0.6 \\
\hline 5.32 & Double bond fatty acid & 18 & -0.55 & -0.6 \\
\hline
\end{tabular}


Fig.1 Score plot of the PCA model obtained with plasma at SL before (T0) endurance exercise test (red) and ALT before (T0) endurance exercise test (blue).

Fig.2 a) Score plot of the PCA model obtained with plasma at SL before (T0) endurance exercise test (red) and after $60 \mathrm{~min}$ (T60) endurance exercise test (blue) b) Score plot of the OPLS model obtained with plasma at SL before (T0) endurance exercise test (red) and after $60 \mathrm{~min}$ (T60) endurance exercise test.(blue) c) OPLS loading plot at SL. Variations of metabolites are represented using a line plot between 0.5 and $5.5 \mathrm{ppm}$. Positive signals correspond to metabolites at increased concentrations in plasma after exercise (T60). Conversely, negative signals correspond to metabolites present at increased concentrations in plasma before exercise (T0). The colours correspond to the $\mathrm{r}$ correlation coefficient. The buckets are labelled according to metabolite assignments presented in Table 2 .

Fig.3 a) Score plot of the PCA model obtained with plasma at ALT before (T0) endurance exercise test (red) and after $60 \mathrm{~min}$ (T60) endurance exercise test (blue) b) Score plot of the OPLS model obtained with plasma at altitude before (T0) endurance exercise test (red) and after $60 \mathrm{~min}$ (T60) endurance exercise test (blue) c) OPLS loading plot at altitude. Variations of metabolites are represented using a line plot between 0.5 and $5.5 \mathrm{ppm}$. Positive signals correspond to metabolites at increased concentrations in plasma after exercise (T60). Conversely, negative signals correspond to metabolites present at increased concentrations in plasma before exercise (T0). The colours correspond to the $\mathrm{r}$ correlation coefficient. The buckets are labelled according to metabolite assignments presented in Table 2 . 


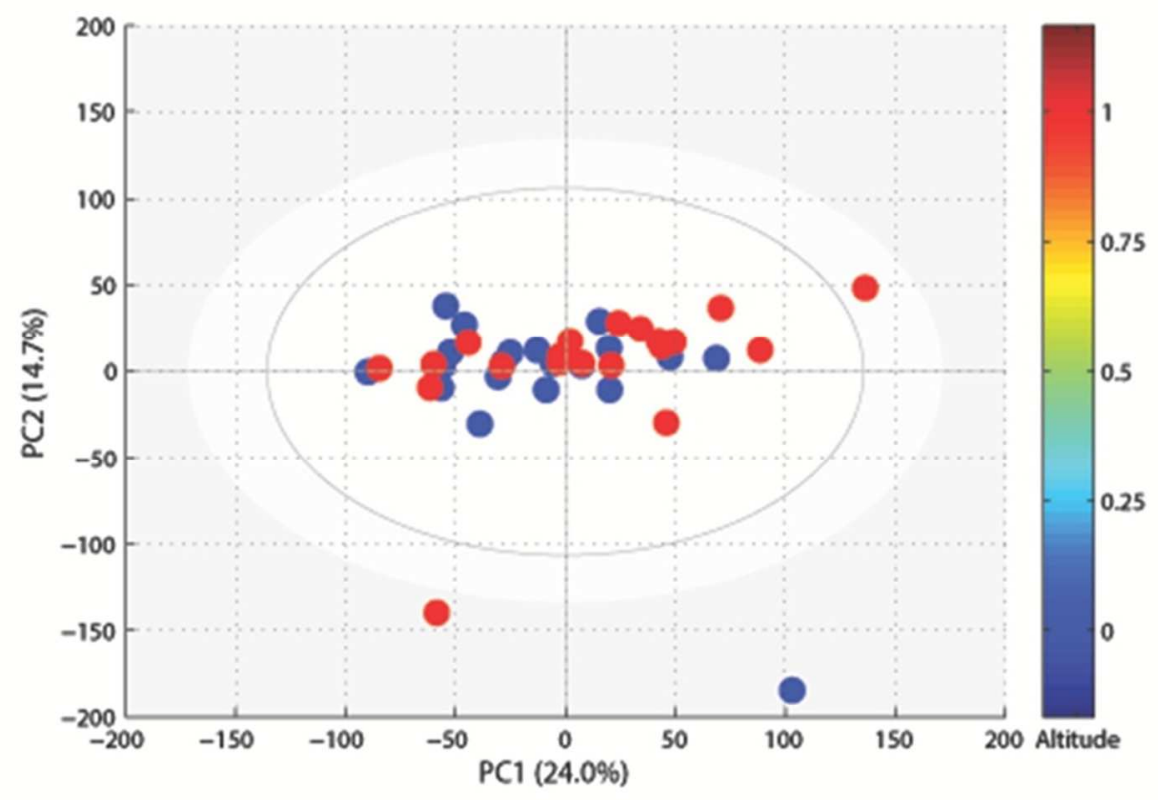

Fig.1 Score plot of the PCA model obtained with plasma at SL before (T0) endurance exercise test (red) and ALT before (TO) endurance exercise test (blue).

$65 \times 43 \mathrm{~mm}(300 \times 300 \mathrm{DPI})$ 

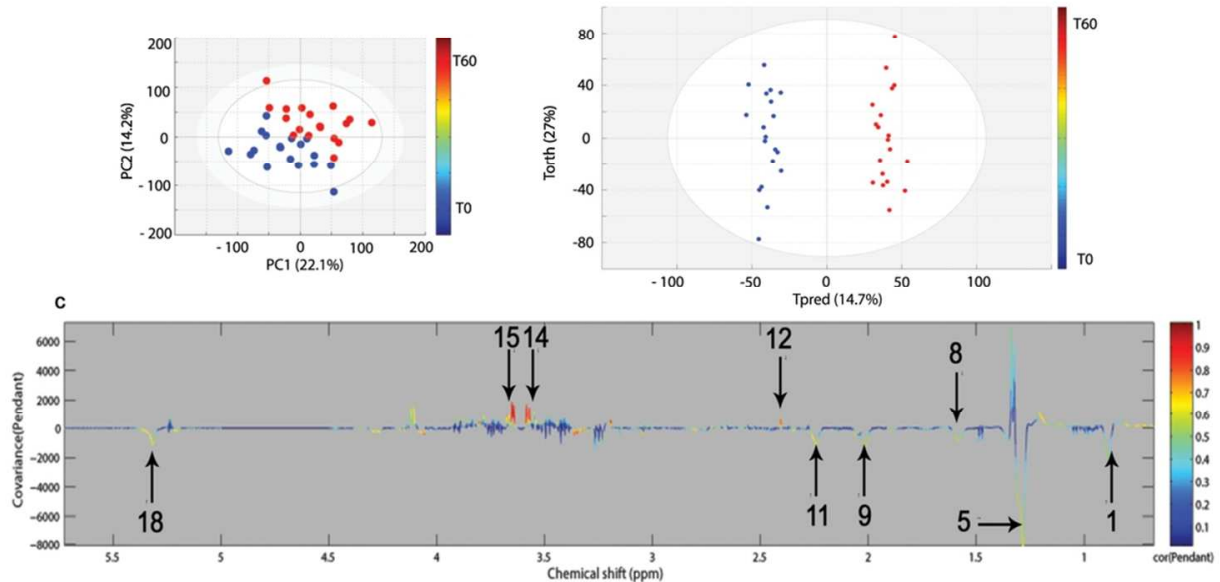

Fig. 2 a) Score plot of the PCA model obtained with plasma at SL before (TO) endurance exercise test (red) and after $60 \mathrm{~min}$ (T60) endurance exercise test (blue) b) Score plot of the OPLS model obtained with plasma at SL before (T0) endurance exercise test (red) and after $60 \mathrm{~min}$ (T60) endurance exercise test. (blue) c) OPLS loading plot at SL. Variations of metabolites are represented using a line plot between 0.5 and 5.5 ppm. Positive signals correspond to metabolites at increased concentrations in plasma after exercise (T60). Conversely, negative signals correspond to metabolites present at increased concentrations in plasma before exercise (TO). The colours correspond to the $r$ correlation coefficient. The buckets are labelled according to metabolite assignments presented in Table 2.

$100 \times 57 \mathrm{~mm}(300 \times 300 \mathrm{DPI})$ 


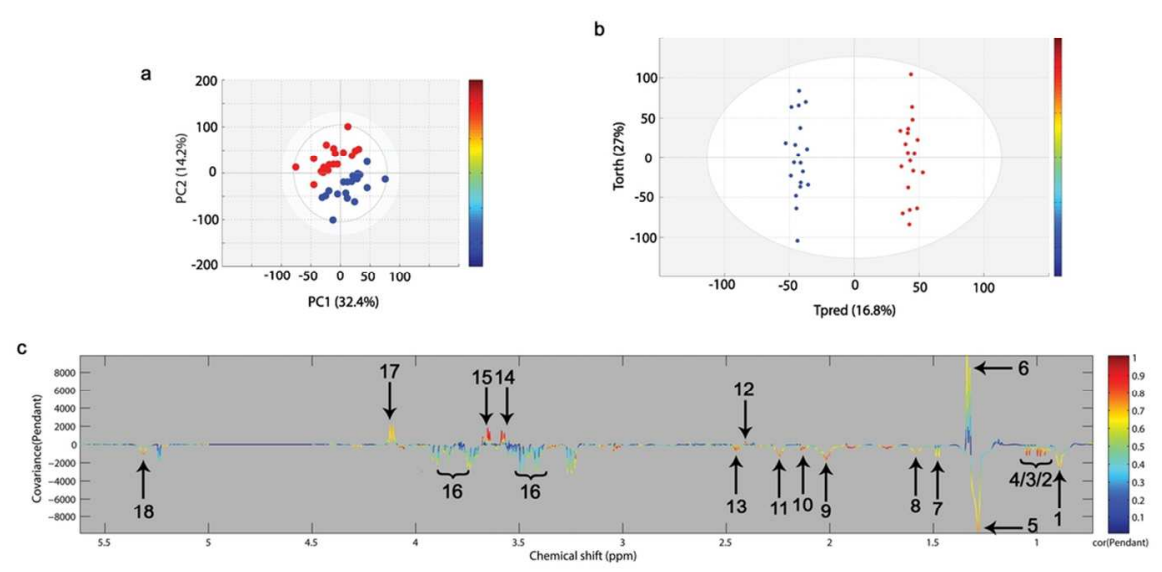

Fig.3 a) Score plot of the PCA model obtained with plasma at ALT before (T0) endurance exercise test (red) and after $60 \mathrm{~min}$ (T60) endurance exercise test (blue) b) Score plot of the OPLS model obtained with plasma at altitude before (TO) endurance exercise test (red) and after $60 \mathrm{~min}$ (T60) endurance exercise test (blue) c) OPLS loading plot at altitude. Variations of metabolites are represented using a line plot between 0.5 and $5.5 \mathrm{ppm}$. Positive signals correspond to metabolites at increased concentrations in plasma after exercise (T60). Conversely, negative signals correspond to metabolites present at increased concentrations in plasma before exercise (TO). The colours correspond to the $r$ correlation coefficient. The buckets are labelled according to metabolite assignments presented in Table 2 .

$103 \times 55 \mathrm{~mm}(300 \times 300 \mathrm{DPI})$ 